\title{
Nonzero Solutions for Nonlinear Systems of Fourth-Order Boundary Value Problems
}

\author{
Ravi P. Agarwal $\mathbb{D}^{1},{ }^{1}$ Mohamed Jleli $\mathbb{D}^{2},{ }^{2}$ and Bessem Samet $\mathbb{D}^{2}$ \\ ${ }^{1}$ Department of Mathematics, Texas A \& M University-Kingsville, Kingsville, TX 78363, USA \\ ${ }^{2}$ Department of Mathematics, College of Science, King Saud University, P. O. Box 2455, Riyadh 11451, Saudi Arabia
}

Correspondence should be addressed to Bessem Samet; bsamet@ksu.edu.sa

Received 9 July 2021; Accepted 10 August 2021; Published 18 August 2021

Academic Editor: Huseyin Isik

Copyright (C) 2021 Ravi P. Agarwal et al. This is an open access article distributed under the Creative Commons Attribution License, which permits unrestricted use, distribution, and reproduction in any medium, provided the original work is properly cited.

This study is devoted to the investigation of nonlinear systems of fourth-order boundary value problems. Namely, using some techniques from matrix analysis and ordinary differential equations, a Lyapunov-type inequality providing a necessary condition for the existence of nonzero solutions is obtained. Next, an estimate involving generalized eigenvalues is derived as an application of our main result.

\section{Introduction}

In this study, we investigate the system of differential equations

$$
\begin{cases}y^{(4)}(t)=\rho(t) \mu(t, y, z), & 0<t<1, \\ z^{(4)}(t)=\sigma(t) \xi(t, y, z), & 0<t<1,\end{cases}
$$

subjected to the boundary conditions

$$
\left\{\begin{array}{l}
y(0)=y^{\prime}(0)=y^{\prime \prime}(1)=y^{(3)}(1)=0, \\
z(0)=z^{\prime}(0)=z^{\prime \prime}(1)=z^{(3)}(1)=0,
\end{array}\right.
$$

where $\rho, \sigma:[0,1] \longrightarrow \mathbb{R}$ and $\mu, \xi:[0,1] \times C([0,1]) \times$ $C([0,1]) \longrightarrow \mathbb{R}$ are the continuous functions with $\mu(\cdot, 0,0)=\xi(\cdot, 0,0) \equiv 0$. Clearly, $(y, z) \equiv(0,0)$ is a trivial solution to (1) and (2). The aim of this study is to obtain necessary conditions for the existence of nonzero solutions to the considered problem. Namely, we establish new Lyapunov-type inequalities [1] for (1) and (2) under reasonable conditions on the nonlinearities $\mu(t, y, z)$ and $\xi(t, y, z)$. Our approach is based essentially on matrix analysis and some arguments from ordinary differential equations.
Fourth-order differential equations are useful in modeling many phenomena from physics ([2-7] and the references therein), which makes the study of such equations particularly interesting. In the literature, several contributions have been devoted to the investigation of sufficient conditions ensuring the existence of solutions to fourth-order boundary value problems ([2, 8-19] and the references therein). The study of necessary conditions for the existence of nontrivial solutions to fourth-order differential equations via Lyapunov-type inequalities has been investigated by some authors [20-22]. For instance, in [20], among other results, it was shown that, if $y$ is a nontrivial solution to

$$
\left\{\begin{array}{l}
y^{(4)}(t)+\lambda(t) y(t)=0, \quad a<t<b, \\
y(a)=y^{\prime}(a)=y^{\prime \prime}(a)=y^{\prime \prime}(b)=0,
\end{array}\right.
$$

where $\lambda:[a, b] \longrightarrow \mathbb{R}$ is continuous, then

$$
\int_{a}^{b} \lambda^{+}(s) \mathrm{d} s>\frac{512}{9(b-a)^{3}},
$$

where $\lambda^{+}(t)=\max \{\lambda(t), 0\}$.

For recent contributions related to Lyapunov-type inequalities, see e.g., [23-29] and the references therein. 
This study is organized as follows. The next section is devoted to some preliminaries. In Section 3, the obtained results as well as their proofs are presented. Finally, some applications to generalized eigenvalue problems are given in Section 4.

\section{Some Preliminaries}

First, we fix some notations. We denote by $\leq_{\mathbb{R}^{2}}$ the partial order in the Euclidean space $\mathbb{R}^{2}$ defined as

$$
\vec{u} \leq_{\mathbb{R}^{2}} \vec{v} \Leftrightarrow u_{i} \leq v_{i}, \quad i=1,2,
$$

for every $\vec{u}=\left(\begin{array}{l}u_{1} \\ u_{2}\end{array}\right), \vec{v}=\left(\begin{array}{l}v_{1} \\ v_{2}\end{array}\right) \in \mathbb{R}^{2}$.

We denote by $M_{2}^{+}$the set of square matrices having nonnegative coefficients, i.e.,

$$
M_{2}^{+}=\left\{\left(m_{i j}\right)_{1 \leq i, j \leq 2}: m_{i j} \geq 0, \quad 1 \leq i, j \leq 2\right\} .
$$

For $C \in M_{2}^{+}$, the trace of $C$ is denoted by Trace $(C)$, the determinant of $C$ is denoted by $\operatorname{det}(C)$, and the spectral radius of $C$ is denoted by $\rho_{C}$, i.e.,

$$
\rho_{C}=\max \left\{\left|\lambda_{i}(C)\right|: i=1,2\right\},
$$

where $\lambda_{i}(C)$ are the complex eigenvalues of $C$.

We equip $M_{2}^{+}$with the norm $\|\cdot\|$ defined as

$$
\|C\|=\sup _{\vec{u} \in \mathbb{R}^{2},\|\vec{u}\|_{2} \neq 0} \frac{\|C \vec{u}\|_{2}}{\|\vec{u}\|_{2}}, \quad C \in M_{2}^{+},
$$

where $\|\cdot\|_{2}$ is the Euclidean norm in $\mathbb{R}^{2}$.

The following lemmas will be useful later.

Lemma 1. Let $\vec{u}, \vec{v} \in \mathbb{R}^{2}$ with

$$
\overrightarrow{0} \leq_{\mathbb{R}^{2}} \vec{u} \leq_{\mathbb{R}^{2}} \vec{v}
$$

where $\overrightarrow{0}$ is the zero vector in $\mathbb{R}^{2}$. Then, $\|\vec{u}\|_{2} \leq\|\vec{v}\|_{2}$.

Proof. The result follows from the fact that

$$
P=\left\{\vec{u} \in \mathbb{R}^{2}: \vec{u} \geq_{\mathbb{R}^{2}} \overrightarrow{0}\right\}
$$

is a normal cone in $\mathbb{R}^{2}$ with normal constant equal to 1 (e.g., [30]).

Lemma 2 (See e.g., [31]). Let $C \in M_{2}^{+}$. If $\rho_{C}<1$, then

$$
\lim _{n \longrightarrow \infty}\|C\|^{n}=0 \text {. }
$$

Lemma 3 (See [25]). Let $C \in M_{2}^{+}$. Then,

$$
\rho_{C}=\frac{\operatorname{Trace}(C)+\sqrt{[\operatorname{Trace}(C)]^{2}-4 \operatorname{det}(C)}}{2} .
$$

Lemma 4 (See [12]). Let $x \in C^{4}((0,1)) \cap C^{1}([0,1)) \cap$ $C^{3}((0,1])$ be a solution to

$$
\left\{\begin{array}{l}
x^{(4)}(t)=f(t), \quad 0<t<1, \\
x(0)=x^{\prime}(0)=x^{\prime \prime}(1)=x^{(3)}(1)=0,
\end{array}\right.
$$

where $f \in C([0,1])$. Then,

$$
x(t)=\int_{0}^{1} H(t, \tau) f(\tau) \mathrm{d} \tau, \quad 0 \leq t \leq 1,
$$

where

$$
0 \leq H(t, \tau)= \begin{cases}\frac{(3 t-\tau) \tau^{2}}{6}, & \text { if } 0 \leq \tau \leq t \leq 1, \\ \frac{(3 \tau-t) t^{2}}{6}, & \text { if } 0 \leq t \leq \tau \leq 1 .\end{cases}
$$

Lemma 5. For all $0<\tau<1$,

$$
\max _{0 \leq t \leq 1} H(t, \tau)=H(1, \tau)=\frac{(3-\tau) \tau^{2}}{6} .
$$

Proof. Fix $0<\tau<1$. Since $H(\cdot, \tau)$ is nondecreasing in $[\tau, 1]$, we deduce that

$$
H(t, \tau) \leq H(1, \tau), \quad \text { for all } t \in[\tau, 1] .
$$

Similarly, it can be easily shown that $H(\cdot, \tau)$ is nondecreasing in $[0, \tau]$, which yields

$$
H(t, \tau) \leq H(\tau, \tau)=\frac{\tau^{3}}{3}, \quad \text { for all } t \in[0, \tau] .
$$

Combining (17) with (18), for all $0 \leq t \leq 1$, we obtain

$$
H(t, \tau) \leq \max \left\{\frac{(3-\tau) \tau^{2}}{6}, \frac{\tau^{3}}{3}\right\}=\frac{(3-\tau) \tau^{2}}{6} .
$$

Hence, (16) is proved.

Throughout this study, we denote by $\|\cdot\|_{\infty}$ the norm in $C([0,1])$ defined as

$$
\|\vartheta\|_{\infty}=\max _{0 \leq t \leq 1}|\vartheta(t)|, \quad \vartheta \in C([0,1]) .
$$

\section{Results and Proofs}

We investigate (1) and (2) under the following assumptions:

(A1) $\rho, \sigma:[0,1] \longrightarrow \mathbb{R}$ are continuous

(A2) $\quad \mu, \xi:[0,1] \times C([0,1]) \times C([0,1]) \longrightarrow \mathbb{R} \quad$ are continuous

(A3) $\mu(\cdot, 0,0)=\xi(\cdot, 0,0) \equiv 0$ (where 0 is the zero function)

(A4) For all $(t, y, z) \in[0,1] \times C([0,1]) \times C([0,1])$,

$$
\begin{gathered}
|\mu(t, y, z)| \leq \mu_{11}(t)\|y\|_{\infty}+\mu_{12}(t)\|z\|_{\infty}, \\
|\xi(t, y, z)| \leq \xi_{21}(t)\|y\|_{\infty}+\xi_{22}(t)\|z\|_{\infty},
\end{gathered}
$$


where $\mu_{11}, \mu_{12}, \xi_{21}, \xi_{22}:[0,1] \longrightarrow[0, \infty)$ are the continuous functions.

By solution to (1) and (2), we mean a pair of functions $(y, z), \quad y, z \in C^{4}((0,1)) \cap C^{1}([0,1)) \cap C^{3}((0,1])$, satisfying (1) and the initial conditions (2). A solution $(y, z)$ to $(1)$ and

(2) is said to be nontrivial, if $(y, z) \equiv(0,0)$.

For $\delta, \eta \in C([0,1])$, let

$$
J_{\delta}(\eta)=\int_{0}^{1}(3-\tau) \tau^{2}|\delta(\tau)| \eta(\tau) \mathrm{d} \tau
$$

Theorem 1. If (1) and (2) have a nontrivial solution, then $J_{\rho}\left(\mu_{11}\right)+J_{\sigma}\left(\xi_{22}\right)+\sqrt{\left(J_{\rho}\left(\mu_{11}\right)-J_{\sigma}\left(\xi_{22}\right)\right)^{2}+4 J_{\sigma}\left(\xi_{21}\right) J_{\rho}\left(\mu_{12}\right)} \geq 12$.

$$
\begin{aligned}
|y(t)| & \leq \int_{0}^{1}|H(t, \tau)\|\rho(\tau)\| \mu(\tau, y, z)| \mathrm{d} \tau \\
& \leq \int_{0}^{1} \frac{(3-\tau) \tau^{2}}{6}|\rho(\tau)|\left(\mu_{11}(\tau)\|y\|_{\infty}+\mu_{12}(\tau)\|z\|_{\infty}\right) \mathrm{d} \tau \\
& =\left(\int_{0}^{1} \frac{(3-\tau) \tau^{2}}{6}|\rho(\tau)| \mu_{11}(\tau) \mathrm{d} \tau\right)\|y\|_{\infty}+\left(\int_{0}^{1} \frac{(3-\tau) \tau^{2}}{6}|\rho(\tau)| \mu_{12}(\tau) \mathrm{d} \tau\right)\|z\|_{\infty}
\end{aligned}
$$

which leads to

$$
\begin{aligned}
\|y\|_{\infty} \leq & \left(\int_{0}^{1} \frac{(3-\tau) \tau^{2}}{6}|\rho(\tau)| \mu_{11}(\tau) \mathrm{d} \tau\right)\|y\|_{\infty} \\
& +\left(\int_{0}^{1} \frac{(3-\tau) \tau^{2}}{6}|\rho(\tau)| \mu_{12}(\tau) \mathrm{d} \tau\right)\|z\|_{\infty} .
\end{aligned}
$$

Similarly, by (A4) and (16), we obtain

$$
\begin{aligned}
\|z\|_{\infty} \leq & \left(\int_{0}^{1} \frac{(3-\tau) \tau^{2}}{6}|\sigma(\tau)| \xi_{21}(\tau) \mathrm{d} \tau\right)\|y\|_{\infty} \\
& +\left(\int_{0}^{1} \frac{(3-\tau) \tau^{2}}{6}|\sigma(\tau)| \xi_{22}(\tau) \mathrm{d} \tau\right)\|z\|_{\infty} .
\end{aligned}
$$

Combining (27) with (28), we deduce that

$$
\overrightarrow{0} \leq_{\mathbb{R}^{2}} \overrightarrow{\phi_{y, z}} \leq \mathbb{R}_{\mathbb{R}^{2}} C \overrightarrow{\phi_{y, z}}
$$

where $\overrightarrow{\phi_{y, z}}=\left(\begin{array}{c}\|y\|_{\infty} \\ \|z\|_{\infty}\end{array}\right)$ and

$$
C=\frac{1}{6}\left(\begin{array}{ll}
J_{\rho}\left(\mu_{11}\right) & J_{\rho}\left(\mu_{12}\right) \\
J_{\sigma}\left(\xi_{21}\right) & J_{\sigma}\left(\xi_{22}\right)
\end{array}\right) \text {. }
$$

Next, using Lemma 3 and (24), we deduce that

$$
\rho_{C}<1 \text {. }
$$

On the other hand, using Lemma 1 and (29), we obtain
Proof. Let $(y, z)$ be a nontrivial solution to (1) and (2), and suppose that

$$
J_{\rho}\left(\mu_{11}\right)+J_{\sigma}\left(\xi_{22}\right)+\sqrt{\left(J_{\rho}\left(\mu_{11}\right)-J_{\sigma}\left(\xi_{22}\right)\right)^{2}+4 J_{\sigma}\left(\xi_{21}\right) J_{\rho}\left(\mu_{12}\right)}<12 .
$$

By Lemma $4,(y, z) \in C([0,1]) \times C([0,1])$ is a nontrivial solution to the system of integral equations:

$$
\left\{\begin{array}{l}
y(t)=\int_{0}^{1} H(t, \tau) \rho(\tau) \mu(\tau, y, z) \mathrm{d} \tau \\
z(t)=\int_{0}^{1} H(t, \tau) \sigma(\tau) \xi(\tau, y, z) \mathrm{d} \tau
\end{array}\right.
$$

Using (A4) and (16), for all $0 \leq t \leq 1$, we obtain 
Obviously, $\mu$ and $\xi$ satisfy (A3). Moreover, for all $(t, y, z) \in[0,1] \times C([0,1]) \times C([0,1])$,

$$
\begin{aligned}
& |\mu(t, y, z)|=|\sin (y(t)+z(t))| \leq|y(t)+z(t)| \leq\|y\|_{\infty}+\|z\|_{\infty}, \\
& |\xi(t, y, z)|=|\arctan (y(t)+z(t))| \leq|y(t)+z(t)| \leq\|y\|_{\infty}+\|z\|_{\infty} .
\end{aligned}
$$

Then, (A4) is satisfied with

$$
\mu_{11}=\mu_{12}=\xi_{21}=\xi_{22}=1
$$

Hence, by Theorem 1, we deduce the following.

Corollary 1. If (35) and (2) have a nontrivial solution, then

$$
J_{\rho}(1)+J_{\sigma}(1)+\sqrt{\left(J_{\rho}(1)-J_{\sigma}(1)\right)^{2}+4 J_{\sigma}(1) J_{\rho}(1)} \geq 12 .
$$

3.2. Nonlocal Source Terms. Consider the system of differential equations

$$
\begin{cases}y^{(4)}(t)=\rho(t) \int_{0}^{t}(t-s)^{\alpha-1}(y(s)+z(s)) \mathrm{d} s, & 0<t<1, \\ z^{(4)}(t)=\sigma(t) \int_{0}^{t}(t-s)^{\beta-1}(y(s)+z(s)) \mathrm{d} s, & 0<t<1,\end{cases}
$$

under the boundary condition (16), where $\rho, \sigma \in C([0,1])$ and $\alpha, \beta>0$. Problem (40) is a particular case of (1) with

$$
\begin{aligned}
& \mu(t, y, z)= \begin{cases}\int_{0}^{t}(t-s)^{\alpha-1}(y(s)+z(s)) \mathrm{d} s, & \text { if } 0<t \leq 1, \\
0, \quad \text { if } t=0,\end{cases} \\
& \xi(t, y, z)= \begin{cases}\int_{0}^{t}(t-s)^{\beta-1}(y(s)+z(s)) \mathrm{d} s & \text { if } 0<t \leq 1, \\
0, \text { if } t=0,\end{cases}
\end{aligned}
$$

for all $y, z \in C([0,1])$. Obviously, $\mu$ and $\xi$ satisfy (A3). Moreover, for all $(t, y, z) \in[0,1] \times C([0,1]) \times C([0,1])$,

$$
\begin{aligned}
|\mu(t, y, z)| & \leq\left(\int_{0}^{t}(t-s)^{\alpha-1} \mathrm{~d} s\right)\left(\|y\|_{\infty}+\|z\|_{\infty}\right) \\
& =\frac{t^{\alpha}}{\alpha}\left(\|y\|_{\infty}+\|z\|_{\infty}\right) .
\end{aligned}
$$

and similarly

$$
|\xi(t, y, z)| \leq \frac{t^{\beta}}{\beta}\left(\|y\|_{\infty}+\|z\|_{\infty}\right) .
$$

Then, (A4) is satisfied with

$$
\begin{aligned}
& \mu_{11}(t)=\mu_{12}(t)=\frac{t^{\alpha}}{\alpha}, \quad 0 \leq t \leq 1, \\
& \xi_{21}(t)=\xi_{22}(t)=\frac{t^{\beta}}{\beta}, \quad 0 \leq t \leq 1 .
\end{aligned}
$$

Then, by Theorem 1, we deduce the following.

Corollary 2. If (39) and (2) have a nontrivial solution, then

$$
J_{\rho}\left(\frac{t^{\alpha}}{\alpha}\right)+J_{\sigma}\left(\frac{t^{\beta}}{\beta}\right)
$$

$$
+\sqrt{\left(J_{\rho}\left(\frac{t^{\alpha}}{\alpha}\right)-J_{\sigma}\left(\frac{t^{\beta}}{\beta}\right)\right)^{2}+4 J_{\sigma}\left(\frac{t^{\beta}}{\beta}\right) J_{\rho}\left(\frac{t^{\alpha}}{\alpha}\right)} \geq 12
$$

Example 1. Consider the system of differential equation (35) with $\rho \equiv 1$ and $\sigma(t)=9 / 4(3-t)$ for all $t \in[0,1]$. Elementary calculations show that

$$
\begin{aligned}
J_{\rho}(1)= & J_{\sigma}(1)=\frac{3}{4}, \\
& J_{\rho}(1)+J_{\sigma}(1)+\sqrt{\left(J_{\rho}(1)-J_{\sigma}(1)\right)^{2}+4 J_{\sigma}(1) J_{\rho}(1)}=3<12 .
\end{aligned}
$$

Hence, by Corollary 1, we deduce that (35) and (2) have no nontrivial solution.

\section{Generalized Eigenvalues Problems}

We say that $e=\left(e_{i j}\right)_{1 \leq i, j \leq 2}$ is a generalized eigenvalue of the system of differential equations

$$
\begin{cases}y^{(4)}(t)=e_{11} y(t)+e_{12} z(t), & 0<t<1, \\ z^{(4)}(t)=e_{21} y(t)+e_{22} z(t), & 0<t<1,\end{cases}
$$

subjected to the boundary condition (2), if (2) and (44) admit a nonzero solution $\left(y_{e}, z_{e}\right)$. Notice that $(44)$ is a particular case of (1) with

$$
\begin{gathered}
\rho(t)=1, \mu(t, y, z)=e_{11} y(t)+e_{12} z(t), \\
(t, y, z) \in[0,1] \times C([0,1]) \times C([0,1]), \\
\sigma(t)=1, \xi(t, y, z)=e_{21} y(t)+e_{22} z(t), \\
(t, y, z) \in[0,1] \times C([0,1]) \times C([0,1]) .
\end{gathered}
$$




$$
\begin{aligned}
& \mu_{11}=e_{11}, \\
& \mu_{12}=e_{12}, \\
& \xi_{21}=e_{21}, \\
& \xi_{22}=e_{22} .
\end{aligned}
$$

Hence, by Theorem 1 , if $e$ is a generalized eigenvalue of (2) and (44), then

$$
J_{1}\left(e_{11}\right)+J_{1}\left(e_{22}\right)+\sqrt{\left(J_{1}\left(e_{11}\right)-J_{1}\left(e_{22}\right)\right)^{2}+4 J_{1}\left(e_{21}\right) J_{1}\left(e_{12}\right)} \geq 12 .
$$

On the other hand, for $\lambda \in \mathbb{R}$, we have

$$
J_{1}(\lambda)=\lambda \int_{0}^{1}(3-\tau) \tau^{2} \mathrm{~d} \tau=\frac{3 \lambda}{4} .
$$

Hence, (50) reduces to

$$
e_{11}+e_{22}+\sqrt{\left(e_{11}-e_{22}\right)^{2}+4 e_{21} e_{12}} \geq 16
$$

Therefore, the following result follows.

Corollary 3. If $e=\left(e_{i j}\right)_{1 \leq i, j \leq 2}$ is a generalized eigenvalue of (43) and (2), then

$$
e_{11}+e_{22}+\sqrt{\left(e_{11}-e_{22}\right)^{2}+4 e_{21} e_{12}} \geq 16
$$

\section{Conclusion}

Using some techniques from matrix analysis and ordinary differential equations, a necessary condition for the existence of nonzero solutions to (1) and (2) is obtained (Theorem 1). As particular cases of (1), we discussed nonlinearities involving trigonometric functions (Corollary 1) and nonlocal source terms (Corollary 2). Finally, we applied our main result to obtain an estimate involving generalized eigenvalues (Corollary 3).

\section{Data Availability}

No data were used to support this study.

\section{Conflicts of Interest}

The authors declare that there are no conflicts of interest.

\section{Acknowledgments}

The third author was supported by Researchers Supporting Project number, King Saud University, Riyadh, Saudi Arabia (RSP-2021/4).

\section{References}

[1] A. Lyapunov, "Problème gńéral de la stabilité du mouvement," Annales de la Faculté des Sciences de Toulouse, vol. 9, pp. 204-474, 1907.

[2] R. P. Agarwal, "On fourth-order boundary value problems arising in beam analysis," Differential and Integral Equations, vol. 2, pp. 91-110, 1989.
[3] P. B. Bailey and S. M. Davenport, "A fourth order nonlinear two point boundary value problem arising in linear elasticity," Applied Mathematics and Computation, vol. 1, no. 4, pp. 333-340, 1975.

[4] N. Y. Golovina, "The nonlinear stress-strain curve model as a solution of the fourth order differential equation," International Journal of Pressure Vessels and Piping, vol. 189, Article ID 104258, 2021.

[5] J. K. Hale, Theory of Functional Differential Equations, Springer-Verlag, New York, NY, USA, 1977.

[6] Y. Wang, Some fourth order differential equations modeling suspension bridges, Ph.D. Thesis, Department of Mathematics, Politecnico Di Milano, Milano, Italy, 2015.

[7] H. Zhong, "Spline-based differential quadrature for fourth order differential equations and its application to Kirchhoff plates," Applied Mathematical Modelling, vol. 28, no. 4, pp. 353-366, 2004.

[8] A. R. Aftabizadeh, "Existence and uniqueness theorems for fourth-order boundary value problems," Journal of Mathematical Analysis and Applications, vol. 116, no. 2, pp. 415-426, 1986.

[9] E. Alves, T. F. Ma, and M. L. Pelicer, "Monotone positive solutions for a fourth order equation with nonlinear boundary conditions," Nonlinear Analysis: Theory, Methods \& Applications, vol. 71, no. 9, pp. 3834-3841, 2009.

[10] A. Cabada and R. R. Enguiça, "Positive solutions of fourth order problems with clamped beam boundary conditions," Nonlinear Analysis: Theory, Methods \& Applications, vol. 74, no. 10, pp. 3112-3122, 2011.

[11] M. Jleli and B. Samet, "Existence and uniqueness of positive solution to a fourth order two point boundary value problem," The Electronic Journal of Differential Equations, vol. 2013, pp. 1-10, 2013.

[12] S. Padhi, "A note on the eigenvalue criteria for positive solutions of a cantilever beam equation with free end," Bulletin of the Iranian Mathematical Society, 2020, In press.

[13] B. Samet, "Existence results for a coupled system of nonlinear fourth-order differential equations," Abstract and Applied Analysis, vol. 2013, Article ID 324848, 9 pages, 2013.

[14] L. Sanchez, "Boundary value problems for some fourth order ordinary differential equations," Applicable Analysis, vol. 38, no. 3, pp. 161-177, 1990.

[15] AK. Verma, B. Pandit, and R. P. Agarwal, "Existence and nonexistence results for a class of fourth-order coupled singular boundary value problems arising in the theory of epitaxial growth," Mathematical Methods in the Applied Sciences, pp. 1-34, 2020, In press.

[16] Y. Wei, Q. Song, and Z. Bai, "Existence and iterative method for some fourth order nonlinear boundary value problems," Applied Mathematics Letters, vol. 87, pp. 101-107, 2019.

[17] Q. Wang and Y. Lu, "Positive solutions for a nonlinear system of fourth-order ordinary differential equations," The Electronic Journal of Differential Equations, vol. 45, pp. 1-15, 2020.

[18] M. Xu and R. Ma, "Nonlinear elastic beam problems with the parameter near resonance," Open Mathematics, vol. 16, no. 1, pp. 1176-1186, 2018.

[19] M. Younis, D. Singh, and A. Petrusel, "Applications of graph Kannan mappings to the damped spring-mass system and deformation of an elastic beam," Discrete Dynamics in Nature and Society, vol. 2019, Article ID 1315387, 9 pages, 2019.

[20] M. F. AktaŞ and D. Çakmak, "Lyapunov-type inequalities for fourth-order boundary value problems," RACSAM, vol. 113, pp. 615-625, 2019. 
[21] S. H. Saker, "Lyapunov's type inequalities for fourth-order differential equations," Abstract and Applied Analysis, vol. 2012, Article ID 795825, 25 pages, 2012.

[22] X. Yang, "On Liapunov-type inequality for certain higherorder differential equations," Applied Mathematics and Computation, vol. 134, no. 2-3, pp. 307-317, 2003.

[23] R. P. Agarwal, M. Bohner, and A. Özbekler, Lyapunov Inequalities and Applications, Springer, Berlin, Germany, 2021.

[24] R. P. Agarwal, M. Jleli, and B. Samet, "On De La Vallée Poussin-type inequalities in higher dimension and applications," Applied Mathematics Letters, vol. 86, pp. 264-269, 2018.

[25] M. Jleli, D. O’Regan, and B. Samet, "Lyapunov-type inequalities for coupled systems of nonlinear fractional differential equations via a fixed point approach," Journal of Fixed Point Theory and Applications, vol. 21, no. 45, pp. 1-15, 2019.

[26] M. Jleli and B. Samet, "A Lyapunov-type inequality for a Laplacian system on a rectangular domain with zero Dirichlet boundary conditions," Mathematics, vol. 7, no. 9, p. 850, 2019.

[27] D. O'Regan and B. Samet, "Lyapunov-type inequality for a class of fractional differential equations," Journal of Inequalities and Applications, vol. 247, pp. 1-10, 2015.

[28] J. Rong and C. Bai, "Lyapunov-type inequality for a fractional differential equation with fractional boundary condition," Advances in Differential Equations, vol. 82, pp. 1-10, 2015.

[29] R. Yang, I. Sim, and Y.-H. Lee, "Lyapunov-type inequalities for one-dimensional Minkowski-curvature problems," Applied Mathematics Letters, vol. 91, pp. 188-193, 2019.

[30] D. Guo, Y. J. Cho, and J. Zhu, Partial Ordering Methods in Nonlinear Problems, Nova Science, New York, NY, USA, 2004.

[31] A. I. Perov, "On the Cauchy problem for a system of ordinary differential equations," Pviblizhen. Met. Reshen. Differ. Uvavn.vol. 2, pp. 115-134, 1964. 